Ann. Biol. anim. Bioch. Biophys., 1979, 19 (3 B), 943-947.

\title{
Effet du niveau protéique sur l'utilisation digestive de l'azote chez le veau ruminant
}

\author{
R. GUILHERMET, R. TOULLEC \\ avec la collaboration technique de Marguerite BEAUFILS ef P. DE SAINT JAN. \\ Station de Recherches Zootechniques, I.N.R.A., \\ Ecole Nationale Supérieure Agronomique \\ 65, rue de St-Brieuc, 35042 Rennes Cédex.
}

Summary. Effect of protein level on nitrogen utilization in the ruminant calf.

Fifteen Friesian bull-calves weaned at 6 weeks of age were divided in 3 groups of 5 animals each. They were fed one of three concentrates containing 14.8-17.4 or 21.1 p. 100 crude protein on a dry matter (DM) basis (treatments 15, 17 and 21). The trial was divided in 3 successive 2-week periods, during which the amounts of DM fed were 64,75 and $84 \mathrm{~g} / \mathrm{kg}$ live weight ${ }^{0,75}$.

The apparent digestibility of nitrogen increased with the level of soyabean oil meal supplementation (table 1 ), but it tended to decrease with age when daily dry matter intake rose. The daily maintenance requirements were estimated to be $310 \mathrm{mg}$ apparently digested nitrogen and $3.44 \mathrm{~g}$ intestinal digestible protein $/ \mathrm{kg}^{0,75}$. Metabolic fæcal nitrogen excretion was estimated to be $3.37 \mathrm{~g} / \mathrm{kg} D M$ ingested and endogenous urinary nitrogen excretion $163 \mathrm{mg} / \mathrm{kg}^{0,75}$.

\section{Introduction.}

De nombreux auteurs ont étudié l'influence du taux azoté de l'aliment concentré sur la croissance et le bilan azoté du veau sevré précocement (Stobo et Roy, 1973). Cependant, plus rares sont les résultats concernant les pertes d'azote métabolique fécal ef d'azote endogène urinaire, ainsi que le besoin d'entretien en azote ou en protéines digestibles dans l'intestin (PDI). Le but de ce travail était d'apporter des données complémentaires sur ces différents points en examinant l'effet du niveau azoté de l'aliment concentré sur l'utilisation digestive ef le bilan azoté dans la phase postsevrage.

\section{Matériel et méthodes.}

Quinze veaux mâles Frisons ont été sevrés à l'âge de 6 semaines et maintenus en cages à bilans jusqu'à 10 ou 12 semaines. Une semaine avant le sevrage, ils ont été divisés en 3 lots de 5 animaux qui ont reçu des aliments concentrés contenant 14,8, 17,4 ou 21,1 p. 100 de matières azotées par rapport à la malière sèche (MS) (traite- 
ments 15, 17 ou 21). Le premier aliment concentré comprenait 85 p. 100 d'orge, 8 p. 100 de tourteau de soja, 3 p. 100 de mélasse et 4 p. 100 de condiment minéral vitaminisé ; sa teneur en cellulose était de 4 p. 100/MS. Les 2 autres aliments concentrés ont été préparés en ajoutant au premier 8 ou 16 p. 100 de tourteau de soja $\left(^{1}\right)$. A partir de la septième semaine, l'essai a comporté 3 périodes $\left({ }^{2}\right)$, au cours desquelles les veaux ont reçu au total 68,77 et $88 \mathrm{~g}$ d'aliment $/ \mathrm{kg}$ de poids vifo, $55 / \mathrm{j}$, dont $87,5 \mathrm{p}$. 100 d'aliment concentré et 12,5 p. 100 de foin ( $\left.{ }^{1}\right)$. Lors de chaque période, les fèces ef l'urine ont été collectées pendant 5 jours, après une phase d'adaptation au niveau alimentaire de 9 jours.

\section{Résultats et discussion.}

Les animaux n'ayant pas consommé la totalité des quantités proposées au cours de chacune des périodes de mesure n'ont pas été retenus pour le calcul des bilans.

TABLEAU 1

Effet du régime sur les performances des veaux (6-10 semaines d'âge)

\begin{tabular}{|c|c|c|c|}
\hline Régime & 15 & 17 & 21 \\
\hline $\begin{array}{l}\text { Teneur en matières azotées totales de l'aliment } \\
\text { concentré }(g / \mathrm{kg} \mathrm{MS}) \ldots \ldots \ldots \ldots \ldots \ldots \ldots\end{array}$ & 148 & 174 & 211 \\
\hline 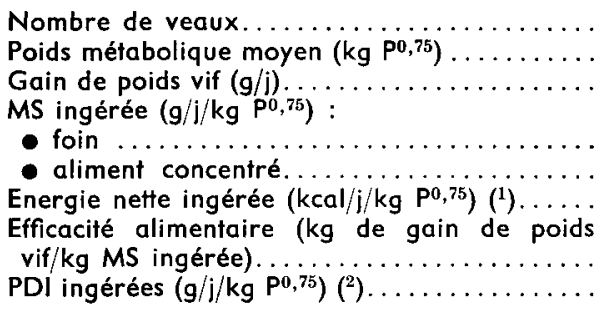 & $\begin{array}{c}5 \\
26,3 \\
653 \\
8,0 \\
60,8 \\
137 \\
\\
0,36 \\
7,23\end{array}$ & $\begin{array}{c}4 \\
25,7 \\
689 \\
\\
8,0 \\
61,7 \\
135 \\
\\
0,37 \\
8,38\end{array}$ & $\begin{array}{c}4 \\
26,9 \\
775 \\
7,8 \\
61,3 \\
131 \\
\\
0,39 \\
9,07\end{array}$ \\
\hline 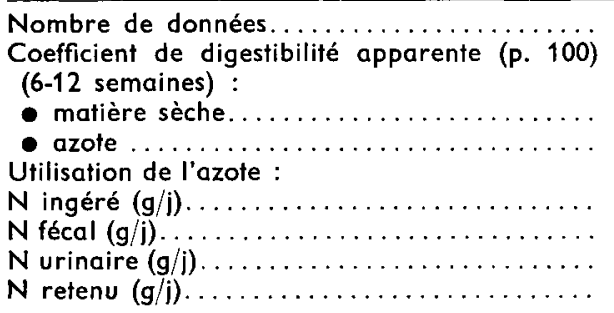 & $\begin{array}{l}77,4 \pm 3,3 \\
70,4\left(^{a}\right) \pm 2,1 \\
42,9\left(^{a}\right) \pm 5,9 \\
12,7 \pm 2,8 \\
10,7\left({ }^{a}\right) \pm 1,6 \\
19,5\left(^{a}\right) \pm 4,3\end{array}$ & $\begin{array}{l}75,7 \pm 2,4 \\
71,8\left({ }^{b}\right) \pm 3,4 \\
\\
47,2\left({ }^{a}\right) \pm 6,9 \\
13,5 \pm 2,2 \\
12,0\left({ }^{b}\right) \pm 3,2 \\
21,8 \pm 6,1\end{array}$ & $\begin{array}{l}75,6 \pm 2,2 \\
74,8\left(a^{a b}\right) \pm 2,7 \\
60,2(a) \pm 6,9 \\
15,1 \pm 1,5 \\
18,1\left(a^{a b}\right) \pm 3,0 \\
26,9(a) \pm 5,4\end{array}$ \\
\hline
\end{tabular}

$(a b)$ : les valeurs de la même ligne, accompagnées des mêmes lettres, sont différentes $(P<0,05)$.

( $\left.{ }^{1}\right)$ L'énergie métabolisable a été calculée en utilisant les équations de Schiemann ef al. (1976). Le rendement de l'énergie méłabolisable en énergie nette a été calculé d'après Vermorel (1978) en utilisant un niveau de production de 1,5 .

$\left({ }^{2}\right)$ Les PDI (protéines digestibles dans l'intestin) ont été calculées à partir des données de Jarrige, Journet et Vérité (1978).

( $\left.{ }^{1}\right)$ Teneur en matières azotées (p. 100 de MS) :54,1 et 9,2 respectivement pour le tourteau de soja et le foin.

(2) Deux veaux par régime seulement ont été utilisés pour la troisième période de mesures. 
Ainsi, les quantités de matière sèche ef d'énergie ingérées n'ont pas été différentes suivant le régime pour chacune des périodes considérées (tabl. 1) ; le gain de poids vif et l'efficacité alimentaire ont eu tendance à augmenter avec le niveau azoté.

Les coefficients de digestibilité de la matière sèche, de la matière organique et de l'azote ont été légèrement (NS) plus élevés à 8 qu'à 10 ou 12 semaines d'âge $(78,2$ p. $100 \pm 1,4-75,3 \pm 2,1$ et $74,8 \pm 3,5$ respectivement pour la matière sèche ; $79,7 \pm 1,4-75,7 \pm 3,7$ ef $76,6 \pm 3,3$ pour la matière organique ; $72,8 \pm 2,8-72,3 \pm 2,3$ et $71,8 \pm 3,5$ pour l'azote). Cette diminution de la digestibilité avec l'âge est vraisemblablement due à l'augmentation du niveau d'ingestion. Elle peut être aussi liée à l'augmentation plus importante du contenu digestif et en particulier du volume du liquide ruminal qui s'effectue immédiatement après le sevrage (Guilhermet et al., 1973). L'absence d'interaction significative entre les périodes et les régimes et de différence significative entre les périodes a permis de rassembler les résultats de digestibilité des 3 périodes afin d'étudier l'effet du régime. La digestibilité apparente des matières azotées a été d'autant plus élevée que la supplémentation en tourteau de soja a été plus forte (tabl. 1). Ceci s'accorde avec les résultats de Brown et al. (1958) et de Stobo et Roy (1973).

L'excrétion d'azote urinaire a été d'autant plus importante $(P<0,01)$ que la teneur en matières azotées des régimes a été plus élevée et que le niveau d'alimentation a été plus fort. Il en a été de même pour la quantité d'azote retenu (tabl. 1).

Les relations entre la quantité d'azote apparemment digéré (NAD, $g / j)$ et celle d'azote retenu $(N R, g / j)$ pour les différents régimes ont été linéaires $(P<0,01)$. Elles n'ont pas été mieux décrites par une régression curvilinéaire, ou par l'expression des données en fonction du poids métabolique. La figure 1 montre la relation pour le régime 15. Puisque la pente de la droite de régression enregistrée avec ce régime $(0,91)$ a été supérieure $(P<0,05)$ à celle obtenue avec les régimes $17(0,54)$ ou $21(0,67)$, ef que la quantité d'azote retenu (calculé après ajustement) a été significativement plus faible (fig. 1), cette droite de régression peut être considérée comme indiquant la réponse de la rétention azotée à l'augmentation de lo quantité d'azote apparemment

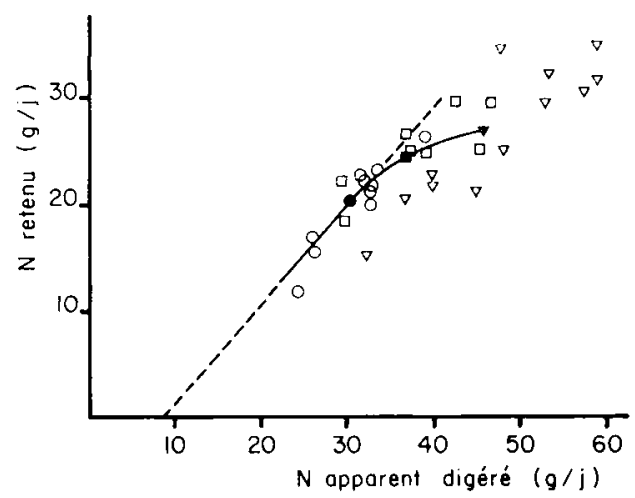

FIG. 1. - Relation entre l'azole apparemment digéré ef l'azote refenu chez les veaux recevant les aliments : 0,$15 ; 0,17 ; \triangle, 21$. La droite de régression (NR $=0,907 \mathrm{NAD}-7,69, \mathrm{R}^{2}=0,83$ ) représente la relation pour le régime 15, lorsque l'azote ingéré est limitant. $\bullet$, a et $\Delta$ montrent les valeurs moyennes de l'azote retenu et de l'azote apparemment digéré. 
digéré lorsque celle d'azote ingéré est limitante. L'extrapolation de cette droite pour une rétention azotée nulle permet d'estimer le besoin d'entretien en azote apparemment digestible à $7,70 \mathrm{~g} / \mathrm{j}$ soit $310 \mathrm{mg} / \mathrm{kg} \mathrm{P} P^{0,75} / \mathrm{j}$. Cette valeur est très voisine de celle observée par Stobo ef Roy (1973), avec des animaux pluss lourds, recevant des régimes similaires mais ingérant davantage d'énergie et réalisant un croît supérieur. La relation entre la quantité de PDI ingérées (PDI, g/j) et celle d'azote retenu $(N R, g / j)$ est décrite par une équation curvilinéaire (PDI $=92,84+3,32 \mathrm{NR}+0,10(\mathrm{NR})^{2}$; $\left.R^{2}=0,77 ; P<0,01\right)$. L'extrapolation de cette courbe pour une rétention azotée nulle indique que le besoin d'entretien en PDI est de $93 \mathrm{~g} / \mathrm{j}$, soit $3,44 \mathrm{~g} / \mathrm{kg} \mathrm{P}, 75 / \mathrm{j}$. Cette valeur est très voisine de celle calculée par Troccon et al. (1978) par la méthode factorielle.

Une relation linéaire a été observée entre le logarithme de l'azote urinaire $(\log N u, g / j)$ et l'azote ingéré (NI, g/j). L'absence de différence significative entre les pentes et la position des droites de régression, correspondant à l'effet du régime sur l'excrétion urinaire, a permis d'établir une relation de la forme :

$$
\log \mathrm{Nu}=0,0093 \mathrm{NI}+0,6439 ; \mathrm{R}^{2}=0,61(\mathrm{P}<0,01) .
$$

L'ordonnée à l'origine de cette droite $(4,4 \mathrm{~g} / \mathrm{j}$ d'azote urinaire) fournit une estimation de l'azote urinaire endogène. Cette valeur est de $163 \mathrm{mg} \mathrm{N} / \mathrm{kg} \mathrm{P0,75}$; elle s'accorde avec celle proposée en 1965 par l'ARC (165 mg) pour des animaux de poids équivalent $(75 \mathrm{~kg})$. Elle est inférieure de 18 p. 100 à celle obtenue par Stobo et Roy (1973) avec des animaux plus lourds, ingérant davantage d'énergie et réalisant des croîts supérieurs.

La relation entre l'azote apparemment digéré (NAD/kg MSI) et l'azote ingéré $(\mathrm{NI} / \mathrm{kg} \mathrm{MSI}$ ) pour l'ensemble des valeurs obtenues est de la forme :

$$
\mathrm{NAD}=0,845 \mathrm{NI}-3,37 ; \mathrm{R}^{2}=0,94(\mathrm{P}<0,001) .
$$

Puisqu'il n'y a pas de différence significative entre les pentes des relations obtenues pour les différents régimes, cette équation indique que le coefficient de digestibilité réelle des protéines a été de 84,5 p. 100. L'extrapolation de la droite de régression oblenue pour une valeur de NI nulle, permet d'estimer l'azote fécal endogène à $3,37 \mathrm{~g} \mathrm{~d}$ 'N $/ \mathrm{kg} \mathrm{MSI}$. Cette valeur est en accord avec celles trouvées par Harris et Loosli (1944) $(3,7 \mathrm{~g})$ et par Stobo et Roy (1973) $(3,43 \mathrm{~g})$. En revanche, elle est inférieure à celle proposée par l'ARC (1965) (5 g), ce qui peut s'expliquer par le fait que l'azote métabolique fécal est d'autant plus élevé que la teneur en cellulose du régime est plus forte (Stobo, 1964) ; or les régimes utilisés dans notre essai étaient très riches en aliment concentré (87,5 p. 100).

En conclusion, chez le veau sevré précocement, la digestibilité apparente des aliments a tendance à diminuer pendant les 6 semaines qui suivent le sevrage, au fur et à mesure que les quantités ingérées augmentent. Le besoin journalier d'entretien peut être estimé à $310 \mathrm{mg}$ d'azote apparemment digestible et à $3,44 \mathrm{~g}$ de $\mathrm{PDI} / \mathrm{kg} \mathrm{P0,75}$; les pertes d'azote métabolique fécal et d'azote endogène urinaire sont respectivement voisines de $3,37 \mathrm{~g} / \mathrm{kg} \mathrm{MSI}$ ef de $163 \mathrm{mg} / \mathrm{kg} \mathrm{P} \mathrm{P}^{0,75}$.

Commission CNERNA Digestion-Absorption/Association des Physiologistes, Paris 5-6 ocfobre 1978. 


\section{Références}

A.R.C. Agriculfural Research Council, 1965. The nutrient requirements of farm livestock. No 2 Ruminants. London Agric. Res. Counc.

BROWN L. D., LASSISTER C. A., EVERETT J. P., SEATH D. M., RUST J. W., 1958. Effect of protein level in calf starters on the growth rate and metabolism of young calves. J. Dairy Sci., 41, 1425-1433.

GUILHERMET R., GIOVANNI R., TOULLEC R., MATHIEU C.-M., 1973. Le sevrage précoce du veau à l'herbe. I. Evolution des phénomènes digestifs. Sci. Agr. Rennes, 123-133.

HARRIS L. T., LOOSLI J. K., 1944. The minimum protein requirement of young Holstein calves. J. Dairy Sci., 27, 650-651.

JARRIGE R., JOURNET M., VERITE R., 1978. Azote, 89-128. In Alimentation des ruminants. Ed. I.N.R.A. Publications, Versailles.

SCHIEMANN R., JENTSH W., WITTENBURG H., HOOFFMANN L., 1976. Die Verwertung der Futterenergie durch waschende. Arch. Tierernähr., 26, 491-517.

STOBO I. J. F., 1964. Studies in the nutrition of young cattle with special reference to rumen development and protein requirements of early weaned calf. Ph. D. Thesis, Univ. Reading.

STOBO I. J. F., ROY J. H. B., 1973. The protein requirement of the ruminant calf. 4. Nitrogen balance studies on rapidly growing calves given diets of different protein content. Br. J. Nutr., 30 , 113-125.

TROCCON J. L., GUILHERMET R., JOURNET M., GEAY Y., 1978. VeauX, 275-295. In Alimentation des ruminants, Ed. I.N.R.A. Publications, Versailles.

VERMOREL M., 1978. Energie, 47-48. In Alimentation des ruminants, Ed. I.N.R.A. Publications, Versailles, 